\title{
ARMAZENAMENTo dA POLPA DE ACERola EM PÓ A TEMPERATURA AMBIENTE ${ }^{1}$
}

\author{
Patrícia M. de A. GOMES², Rossana M. F. de FIGUEIRÊDO ${ }^{3, *}$, Alexandre J. de M. QUEIROZ ${ }^{3}$
}

\begin{abstract}
RESUMO
Este trabalho foi realizado com o objetivo de se estudar o armazenamento da polpa de acerola em pó embalada em sacos de polietileno durante 60 dias, sob atmosfera ambiente. O pó de acerola foi obtido mediante a secagem de uma solução contendo $90 \%$ de polpa de acerola e $10 \%$ de maltodextrina, em um secador do tipo leito de jorro, com temperatura do ar de secagem de $70^{\circ} \mathrm{C}$. A cada 10 dias foram feitas avaliações dos parâmetros ácido ascórbico, umidade, $\mathrm{pH}$ e cor. Os resultados demonstraram uma diminuição no teor de ácido ascórbico de 29,72\%; um aumento de 51,31\% na umidade; o valor do $\mathrm{pH}$ permaneceu praticamente inalterado oscilando entre 3,7 e 3,8 unidades de $\mathrm{pH}$ e observou-se alterações na cor do pó de acerola ao final do armazenamento, com acréscimo nos valores da intensidade de vermelho e amarelo e diminuição da luminosidade.

Palavras-chave: Malphighia emarginata D.C; leito de jorro; conservação.
\end{abstract}

\section{SUMMARY}

STORAGE OF THE PULP OF POWDERED WEST INDIAN CHERRY THE TEMPERATURE ADAPTS. This work had for objective, to study the storage of the pulp of powdered West Indian cherry wrapped in sacks of polyethylene for 60 days, under atmosphere it adapts. The West indian cherry powder was obtained by the drying of a solution containing $90 \%$ of West Indian cherry pulp and $10 \%$ of maltodextrin, in a dryer of the type spouted bed, with temperature of the air of drying of $70^{\circ} \mathrm{C}$. Every 10 days were made evaluations of the parameters ascorbic acid, humidity, $\mathrm{pH}$ and color. The results a decrease in the tenor of ascorbic acid of $29.72 \%$; an increase of $51.31 \%$ in the humidity; the value of the $\mathrm{pH}$ stayed practically unaffected oscillating between 3.7 and 3.8 units of pH and it was observed alterations in the color of the West Indian cherry powder at the end of the storage, with increment in the values of the intensity of red and yellow and decrease of the brightness

Keywords: Malphighia emarginata D.C.; spouted bed; storage.

\section{1 - INTRODUÇÃO}

Nos últimos anos a acerola (Malphighia emarginata, D.C.) vem sendo explorada comercialmente, com boa aceitação no mercado devido, especialmente, ao seu elevado teor de ácido ascórbico (vitamina C), bem como as suas características nutricionais, associado a sabor e textura agradáveis ao paladar do consumidor. O teor de ácido ascórbico presente na acerola, em torno de $800 \mathrm{mg} / 100 \mathrm{~g}$ em frutos maduro, $1600 \mathrm{mg} / 100 \mathrm{~g}$ em frutos meio-maduro e $2.700 \mathrm{mg} / 100 \mathrm{~g}$ em frutos verdes [3] chega a ser aproximadamente 100 vezes maior que a encontrada na laranja, ou 10 vezes maior que a da goiaba, tidas como frutas possuidoras de alto conteúdo de vitamina C [9]. O aumento da produção e do consumo da acerola, aliados ao fato de se tratar de um fruto muito perecível, torna premente a necessidade de se desenvolver alternativas para seu processamento, visando tanto a conservação, como a obtenção de produtos com maior valor agregado. Sob esse aspecto a acerola leva vantagem sobre a maioria das frutas devido ao alto teor de ácido ascórbico presente na sua polpa, o que lhe confere possibilidades de industrialização e armazena-

\footnotetext{
Recebido para publicação em 10/02/2003. Aceito para publicação em 29/06/2004 (001061).

2. Curso de Pós- Graduação em Engenharia Agrícola, UFCG.

3. Departamento de Engenharia Agrícola, Universidade Federal de Campina Grande, Av. Aprígio Veloso, 882, Caixa Postal 10017, CEP: 58109970, Campina Grande, PB. E-mail: rossana@deag.ufcg.edu.br * A quem a correspondência deve ser enviada.
}

mento com a manutenção de valores nutricionais ainda elevados.

A secagem é um dos processos disponíveis para a aplicação na indústria de polpas de frutas, concentrando os princípios da matéria-prima e habilitando o produto para o armazenamento em condições ambientais por longos períodos.

A secagem em leito de jorro, inicialmente utilizada para materiais granulares, no recobrimento de partículas e na secagem de grãos, tem evoluído consideravelmente, ocupando importante lugar no processamento de alimentos em substituição ao "spray dryer". Experimentos com o leito de jorro para a secagem de polpa de frutas tem-se ampliado a cada dia, obtendo-se produtos com boa qualidade e baixo custo. Testes foram realizados com polpa de umbu [10], polpa de manga [14], serigüela, acerola e cajá [11], entretanto nesses estudos foi dado ênfase apenas ao desempenho do processo (testes fluidodinâmicos) e a alguns parâmetros sobre a qualidade do produto obtido, o estudo do armazenamento desses produtos não tem sido feito.

Produtos submetidos a secagem, embora se beneficiem do retardo no crescimento de microrganismos e do aumento no tempo de conservação [6], necessitam, nas fases de transporte e armazenamento, de embalagem adequada, que mantenham as características do produto obtidas na secagem. Nestas condições, em se tratando especificamente de ácido ascórbico, sua susceptibilidade à degradação depende, dentre outros fatores, do tempo de armazenamento, e oxigênio [15], que podem ser afetados pelo tipo de embalagem. 
Em produtos alimentícios armazenados o tipo de embalagem empregada influencia as reações metabólicas, diminuindo o seu ritmo por meio da modificação do microclima criado em seu interior e constituindo-se numa barreira que impede ou dificulta o contato entre o ambiente externo e o produto [8].

Devido ao baixo custo e aplicação diversificada, inúmeras opções de embalagens plásticas flexíveis, semirígidas e rígidas, estão disponíveis no mercado, com diferentes características de barreira. Dentre os plásticos mais utilizados e de menor preço em todo o mundo o polietileno é dos mais versáteis, apresentando resistência e flexibilidade que o tornam aplicável a um elevado número de opções de embalagem [5]. As suas principais vantagens são a facilidade de soldagem, baixa permeabilidade ao vapor de água, excelentes características de isolamento elétrico, inércia química (resistente a ácidos, álcalis, solventes orgânicos) e grande resistência à tração [5, 16].

A elaboração de polpa de acerola em pó por um processo de baixo custo e sua embalagem em material de fácil obtenção e preços atrativos constitui-se numa alternativa para o melhor aproveitamento da acerola e do seu potencial nutritivo.

O objetivo deste trabalho foi avaliar a estabilidade da polpa de acerola em pó, produzida pela secagem da polpa fresca em secador do tipo leito de jorro.

\section{2 - MATERIAL E MÉTODOS}

A polpa de acerola em pó, utilizada neste trabalho, foi obtida mediante a secagem de uma solução contendo $90 \%$ de polpa de acerola e $10 \%$ de maltodextrina, em um secador do tipo leito de jorro, com temperatura do ar de secagem de $70^{\circ} \mathrm{C}$. As amostras da polpa de acerola em pó foram embaladas em sacos de polietileno, contendo aproximadamente $10 \mathrm{~g}$, e armazenadas durante 60 dias a temperatura ambiente. A cada 10 dias foram feitas avaliações quanto aos teores de: $\mathrm{pH}$, umidade, ácido ascórbico e cor.

$\mathrm{O}$ pH das amostras reconstituídas com água destilada foi determinado através do método potenciométrico, com peagâmetro da marca Analyser modelo 300 M.

A umidade das amostras foi determinada, utilizando-se cerca de $1 \mathrm{~g}$ da amostra, em estufa a $70^{\circ} \mathrm{C}$ até peso constante.

A determinação do ácido ascórbico foi feita nas amostras reconstituídas com água destilada, de acordo com a metodologia titulométrica da AOAC [2] modificada por BENASSI \& ANTUNES [4] onde substituiu-se a solução de extração de ácido metafosfórico por ácido oxálico.

As medidas do parâmetro cor foram realizadas utilizando-se colorímetro de marca Minolta, modelo CR 10, sendo os resultados expressos no sistema CIELAB de cor, onde $\mathrm{L}^{*}$ indica a luminosidade e $\mathrm{a}^{*} \mathrm{e} \mathrm{b*}$ são as coordenadas de cromaticidade $\left(+\mathrm{a}^{*}=\right.$ vermelho; $-\mathrm{a}^{*}=$ verde; $+b^{*}=$ amarelo; $-b^{*}=$ azul).
A partir dos dados obtidos experimentalmente foi realizada a análise estatística utilizando-se o programa computacional ASSISTAT versão 6.2 [19], em delineamento inteiramente casualizado.

Ajustes aos dados experimentais foram realizados com equações do tipo linear e quadrática, através do programa computacional STATISTICA versão 5.0. Os critérios usados para se determinar o melhor ajuste de cada equação, foram o coeficiente de determinação $\left(\mathrm{R}^{2}\right)$ e o desvio percentual médio $(\mathrm{P})$ calculado pela Equação (1).

$\mathrm{P}=\frac{100}{n} \cdot \sum_{i=1}^{n} \frac{\left|\left(\mathrm{M}_{\exp }-\mathrm{M}_{\text {teor }}\right)\right|}{\mathrm{M}_{\exp }}$

Em que:

$\mathrm{M}_{\exp }=$ valores obtidos experimentalmente

$\mathrm{M}_{\text {teor }}=$ valores preditos pelo modelo

$\mathrm{n}=$ número de dados experimentais

\section{3 - RESULTADOS E DISCUSSÃO}

\section{1 - Ácido ascórbico}

Nos sete períodos de tempo em que foram avaliadas as amostras, foram verificadas diferenças significativas entre os valores médios do ácido ascórbico a nivel de $1 \%$ de probabilidade pelo teste $\mathrm{F}$, para os diferentes tempos de armazenamento.

Tem-se na Figura 1 a representação gráfica dos valores médios experimentais do ácido ascórbico da polpa de acerola em pó, em função do tempo de armazenamento. Constatam-se diferenças entre os valores médios do ácido ascórbico para os diferentes tempos de armazenamento. O teor de ácido ascórbico diminuiu com o aumento do tempo de armazenamento, atingindo um percentual de redução de $29,72 \%$ no final (60 dias) em relação ao inicio.

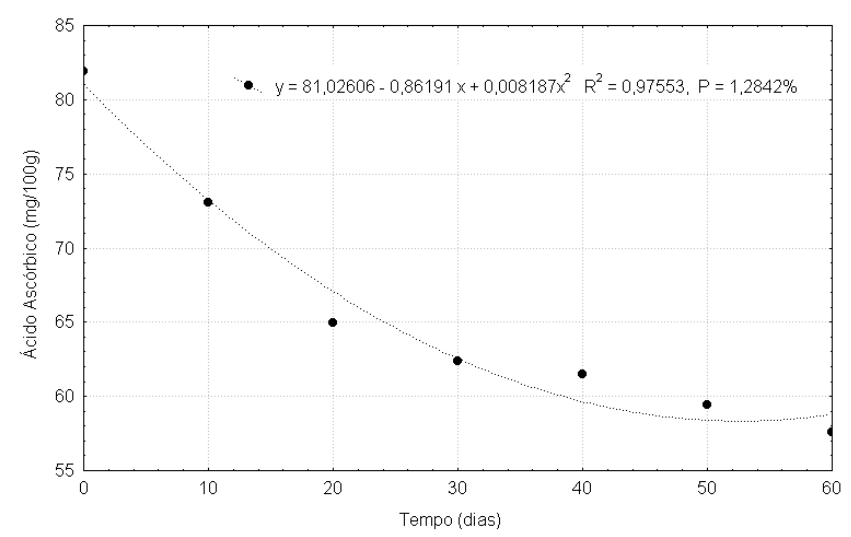

FIGURA 1. Teor de ácido ascórbico (mg/100g) da polpa de acerola em pó, durante o armazenamento.

A curva ajustada aos dados experimentais com a equação quadrática, os parâmetros de ajuste e o desvio percentual médio encontram-se na Figura 1. Nota- 
se pelo valor do coeficiente de determinação $\left(R^{2}\right)$ que a equação quadrática se ajustou bem aos dados experimentais, apresentando $\mathrm{R}^{2}$ acima de 0,97 e o desvio percentual médio de 1,28\%. De acordo com LOMAURO, BAKSHI \& LABUZA [12] um valor de P menor que cinco indica um bom ajuste da equação aos dados experimentais. Dos dados apresentados pode-se ver reduções mais acentuada nos teores de ácido ascórbico nos tempos iniciais do armazenamento, sobretudo no primeiros vinte dias.

Diversos autores já estudaram o efeito do tempo de armazenamento na degradação do ácido ascórbico em frutas e seus produtos, testando vários tipos de equações. ALVES [1] representou o comportamento da vitamina $\mathrm{C}$ da acerola através de equação polinomial de $2^{\circ}$ grau, obtendo um $\mathrm{R}^{2}$ de 0,8640 . Considerando-se que este tipo de equação, quando utilizada para representar as perdas de ácido ascórbico apresentará uma divergência progressiva do processo real a partir do seu ponto de inflexão, sua aplicabilidade restringe-se dentro de determinado intervalo de tempo. No caso presente, esta aplicabilidade é aceitável entre zero e 60 dias (inclusive).

\section{2 - Umidade}

Ao se realizar a análise de variância do teor de umidade da polpa de acerola em pó verificou-se que o parâmetro tempo de armazenamento, com valor de $\mathrm{F}$ igual a 73,4103 apresentou resultado significativo a nivel de $1 \%$, valor este que representa a probabilidade de erro, ao se rejeitar a hipótese de nulidade.

Na Figura 2 são apresentados graficamente os valores obtidos experimentalmente do teor de umidade da polpa de acerola em pó, em função do tempo de armazenamento. O valor do teor de umidade inicial foi de 4,074\%, resultado inferior ao determinado por LIMA et al. [11] que foi de $15,79 \%$ para o pó de acerola desidratado em leito de jorro, nas mesmas condições operacionais. Observa-se também que o teor de umidade aumentou com o tempo, atingindo um percentual de ganho de umidade de $51,31 \%$ ao final do armazenamento (60 dias) em relação ao inicio. Ao estudarem a estabilidade do pó de acerola desidratada pelo processo "FOAM-MAT", durante o armazenamento à temperatura ambiente, SOARES et al. [20] observaram comportamento semelhante, onde trabalharam com um teor de umidade inicial de 7,24\% o qual, após 90 dias, subiu para 12,30\%, resultando num aumento de $69,89 \%$. Este acréscimo na umidade durante o armazenamento é previsível quando a embalagem utilizada não é impermeável ao vapor d'água e a amostra apresenta comportamento higroscópico. FIGUEIRÊDO [7] observou que não houve variação de umidade do pó de acerola acondicionada em embalagem laminada com quatro camadas ao longo dos 360 dias de armazenamento.

Tem-se ainda na Figura 2 a curva da umidade ajustada por equação do tipo linear, os parâmetros de ajuste da equação e o desvio percentual. Interpretando o caráter crescente da umidade com o tempo, até quarenta dias a curva prediz valores inferiores de umidade em relação aos pontos experimentais. Para tempos superiores a cinqüenta dias o ajuste prediz valores superiores aos experimentais. Observa-se que a equação linear apresentou coeficiente de determinação $\left(\mathrm{R}^{2}\right)$ superior a 0,99, ou seja, ajustou-se bem aos dados experimentais, podendo ser usada para predizer o comportamento da umidade com o tempo.

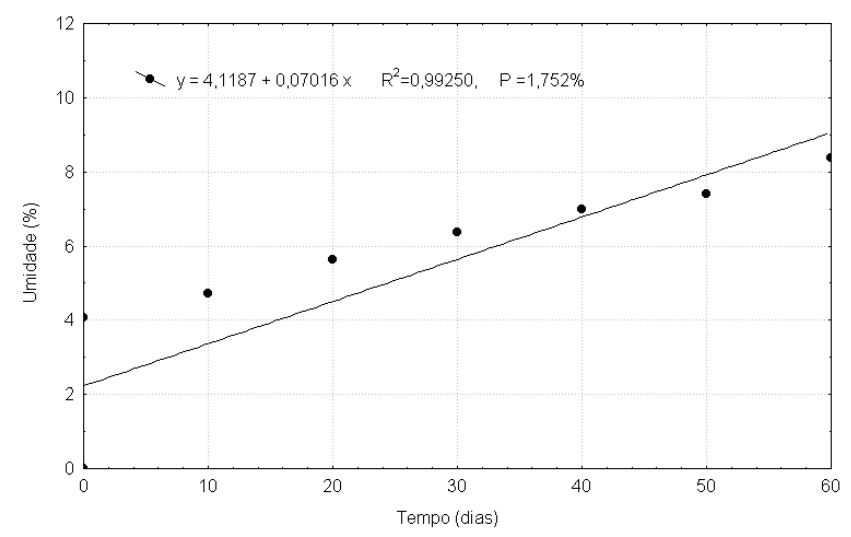

FIGURA 2. Teor de umidade (\%) da polpa de acerola em pó, durante o armazenamento.

\section{$3.3-$ pH}

Pela análise de variância para o $\mathrm{pH}$ da polpa de acerola em pó verificou-se diferença significativa, pelo teste $\mathrm{F}$, para o fator tempo de armazenamento a nível de $1 \%$ de probabilidade ( $\mathrm{p}<0,01 ; \mathrm{F}=9,2120)$.

TABELA 1. Valores médios do $\mathrm{pH}$ da polpa de acerola em pó, durante o armazenamento

\begin{tabular}{cc}
\hline Tempo (dias) & $\mathrm{pH}$ \\
\hline 0 & $3,820 \mathrm{~b}$ \\
10 & $3,826 \mathrm{ab}$ \\
20 & $3,830 \mathrm{ab}$ \\
30 & $3,883 \mathrm{a}$ \\
40 & $3,826 \mathrm{ab}$ \\
50 & $3,760 \mathrm{c}$ \\
60 & $3,843 \mathrm{ab}$
\end{tabular}

$\mathrm{CV}(\%)=3,827 ; \mathrm{DMS}=0,058 ; \mathrm{MG}=3,827$

MG-Média geral, CV-Coeficiente de variação e DMS -Desvio mínimo significativo Obs.: Médias seguidas pela mesma letra não diferem estatisticamente pelo teste Tukey a nivel de $5 \%$ de probabilidade

Na Tabela 1 estão apresentados os valores médios do pH da polpa de acerola em pó, durante o armazenamento. Verifica-se que houve oscilações nos valores médios do $\mathrm{pH}$, entre 3,7 e 3,8 , não ficando evidente nenhum tipo de comportamento padrão. No tempo de 50 dias apresenta-se isoladamente uma diferença significativa com relação a todos os outros períodos, não existindo, entretanto, diferença significativa entre o período inicial e o final. Constata-se que o valor do $\mathrm{pH}$ da polpa de acerola em pó no tempo zero foi 3,82, encontrando-se um pouco acima do obtido por SOARES et al. [20] para a acerola em pó, que foi de 3,22. Em seus 
estudos com a polpa de acerola preservada por três métodos de conservação (congelamento, tratamento térmico e utilização de aditivos químicos) PIMENTEL [17] observou que o pH se manteve estável ao longo de um período de 180 dias. Comparando-se o $\mathrm{pH}$ da polpa antes da secagem, verifica-se que após a transformação em pó ocorreu aumento de aproximadamente $27 \%$.

\section{4 - Cor}

\subsection{1 - Luminosidade ( $\left.L^{*}\right)$}

Da análise de variância dos valores de luminosidade (L*) da polpa de acerola em pó, constatou-se diferença significativa, pelo teste $\mathrm{F}$, entre os tempos de armazenamento, a nível de $1 \%$ de probabilidade $(\mathrm{p}<0,01$; $\mathrm{F}=70,7427$ ).

Estão representados, na Figura 3, os valores médios de L* (luminosidade) da polpa de acerola em pó, em função do tempo de armazenagem. Observa-se diminuição no valor da luminosidade com o aumento do tempo a qual, em termos percentuais e ao final do armazenamento, totalizou $18,84 \%$. As reduções foram gradativas e se mantiveram desde o início até o final do armazenamento. ROBBINS \& MOORE [18] armazenaram frutos de framboesa durante 16 dias nas temperaturas de $0^{\circ} \mathrm{C}$ e $4,5^{\circ} \mathrm{C}$ e durante 8 dias na temperatura de $20^{\circ} \mathrm{C}$, constatando que os parâmetros da cor diminuíram com o tempo. Esse fato foi também observado por OLIVA, MENEZES \& FERREIRA [15] ao armazenarem néctar de acerola (Spin Cooker), porém seus resultados demonstraram que as amostras armazenadas a temperatura ambiente apresentaram diminuição significativa no valor da luminosidade ( $\left.\mathrm{L}^{*}\right)$,com relação ao início apenas a partir de 75 dias.

Os valores de L* (luminosidade) da polpa de acerola em pó, em função do tempo de armazenagem são apresentados na Figura 3, a curva de ajuste do tipo quadrática, bem como os parâmetros de ajuste da equação. Constata-se a boa interpretação dos dados experimentais a partir da curva produzida pelo ajuste da equação, representando adequadamente o comportamento decrescente da luminosidade com o tempo.

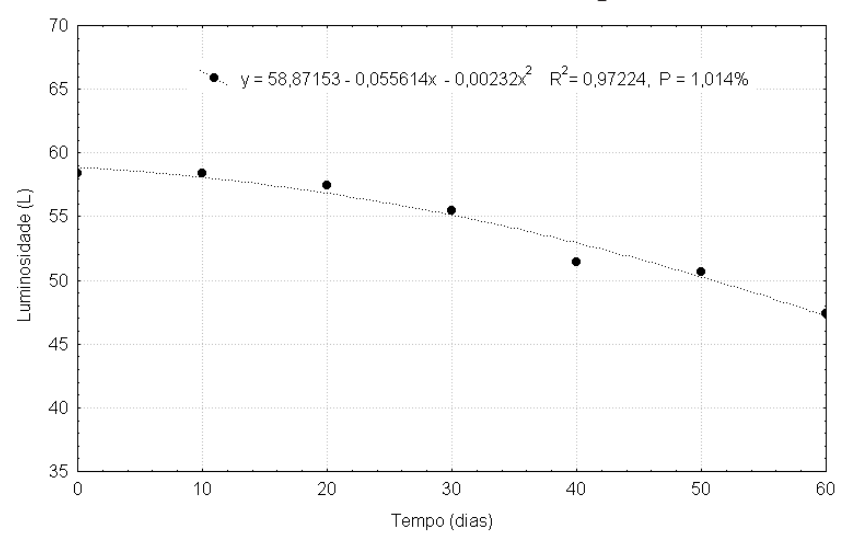

FIGURA 3. Valores do parâmetro luminosidade (L") da polpa de acerola em pó, durante o armazenamento com valores ajustados pela equação quadrática.

\subsection{2 - Intensidade de vermelho $\left(a^{*}\right)$}

A análise de variância revelou efeito significativo $(\mathrm{p} \leq 0,01)$ para o tratamento tempo de armazenamento sobre os resultados da intensidade de vermelho (a*) da polpa de acerola em pó, apresentando um valor de $\mathrm{F}$ (teste F) igual a 17,2353.

Entre o tempo inicial e o final (60 dias) houve um aumento de aproximadamente $35 \%$ na intensidade de vermelho. O aumento da intensidade de vermelho ( $\left.\mathrm{a}^{*}\right)$ associado à redução da luminosidade retratou o escurecimento da amostra. Segundo SOARES et al. [20] podese justificar e correlacionar o aumento da taxa de escurecimento ao decréscimo do teor de ácido ascórbico, possivelmente conseqüência do processo de degradação oxidativa caracterizado como escurecimento não enzimático. Muitos autores têm associado o escurecimento em produtos de frutas com $\mathrm{pH}$ inferior a 4,0 como sendo proveniente da degradação do ácido ascórbico, principalmente anaeróbica, que gera produtos como furfural e polímeros escuros [15]. LIMA et al. [11], por sua vez, observaram que o pó de acerola obtido em leito de jorro apresentou tendência ao escurecimento quando submetido à temperatura do ar de secagem acima de $50^{\circ} \mathrm{C}$.

Na Figura 4 tem-se a curva e os parâmetros de ajuste da equação quadrática utilizada para ajuste dos dados experimentais de intensidade de vermelho (a*) da polpa de acerola em pó, em função do tempo. Ao contrário do parâmetro luminosidade, vê-se um caráter crescente na intensidade de vermelho.

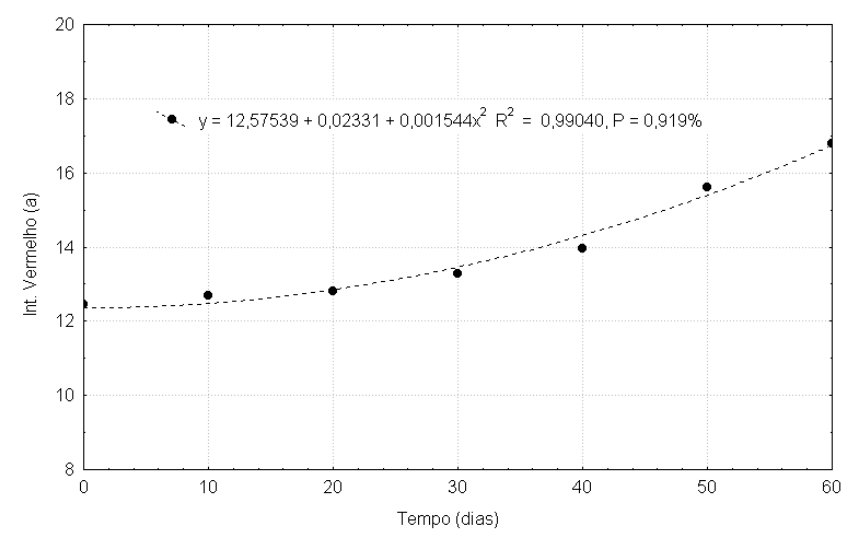

FIGURA 4. Valores de intensidade de vermelho (a*) da polpa de acerola em pó, durante o armazenamento com valores ajustados pela equação quadrática.

\subsection{3 - Intensidade de amarelo $\left(b^{*}\right)$}

Da análise de variância dos valores de intensidade de amarelo (b*) da polpa de acerola em pó, verificou-se que, a nível de significância de $1 \%$, os valores da intensidade de amarelo durante o armazenamento não são, em média, iguais. Não houve diferença significativa entre as médias dos valores de $b^{*}$ entre os quatro primeiros períodos e entre os três últimos períodos de armazenamento. Apesar disso o percentual de aumen- 
to do valor de $\mathrm{b}^{*}$ no final do armazenamento (60 dias) com relação ao tempo zero foi de aproximadamente $21 \%$. Este aumento pode ser devido à reação das antocianinas com o ácido ascórbico presentes na acerola. Desta reação resultariam perdas de ambos os componentes, com formação de pigmentos levemente escuros. Este comportamento é idêntico ao determinado por MATSUURA [13] que também verificou aumento na intensidade de amarelo durante o armazenamento do suco integral de acerola com e sem tratamento térmico, durante 180 dias a $-18^{\circ} \mathrm{C}$. FIGUEIRÊDO [7] que observou um resultado inverso, constatando diminuição na intensidade de amarelo durante o armazenamento do pó de acerola microencapsulado em embalagem laminada.

Na Figura 5, é apresentada a curva e os parâmetros de ajuste da equação quadrática testada para predizer a intensidade de amarelo (b*) da polpa de acerola em pó durante o armazenamento, onde se observa a tendência crescente da variável ao longo do tempo.

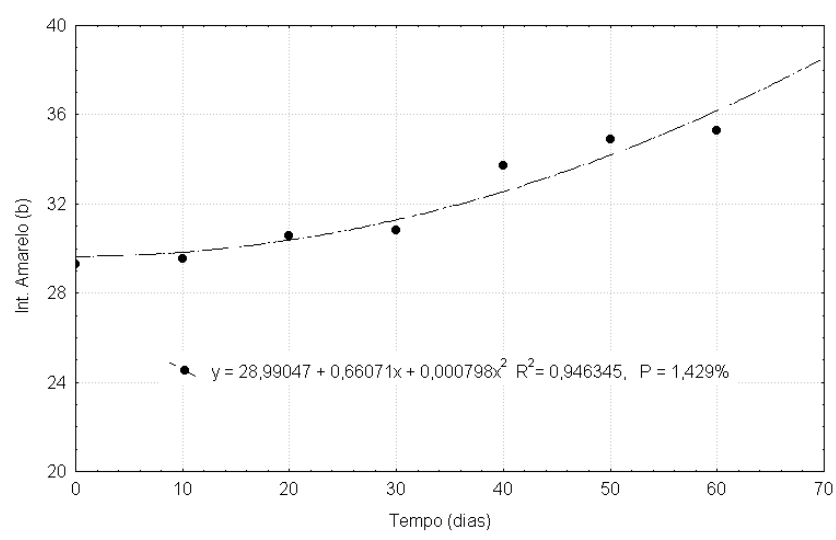

FIGURA 5. Valores de intensidade de amarelo (b) da polpa de acerola em pó, durante o armazenamento por 60 dias com valores ajustados pela equação quadrática.

\section{4 - CONCLUSÕES}

A perda no teor de ácido ascórbico ao final do tempo de armazenagem foi de $29,72 \%$, com as maiores perdas percentuais concentrando-se nos vinte primeiros dias de armazenagem, reduzindo-se nesse período em aproximadamente $20,6 \%$.

$\mathrm{O}$ pH das amostras reconstituídas da acerola em pó apresentou valores entre 3,7 e 3,8, ao longo do armazenamento, demonstrando estabilidade durante o período de tempo estudado.

Os parâmetros relacionados a cor sofreram modificações ao longo do armazenamento, resultado das alterações sofridas pela cor inicial do material durante o período de estudo.

De um modo geral, a embalagem utilizada para armazenar a polpa de acerola em pó não forneceu proteção satisfatória, uma vez que permitiu a troca de umidade do produto com a atmosfera ambiente, não impedindo o escurecimento da amostra.

\section{5 - REFERÊNCIAS BIBLIOGRÁFICAS}

[1] ALVES, R.E. Qualidade de acerola submetida à diferentes condições de congelamento, armazenamento e aplicação pós-colheita de cálcio. Lavras. 1999, 117 p. Tese (Doutorado em Ciência dos Alimentos). Universidade Federal de Lavras (UFLA).

[2] ASSOCIATION OF OFFICIAL ANALYTICAL CHEMISTS. AOAC. Official Methods of Analysis, WILLIAMS, S. (Ed) 14.ed. Arlington, 1984. 1141p.

[3] BATISTA, M.S.; FIGUEIRÊDO, R.M.F.; QUEIROZ, A.J.M. Parâmetros físico-químicos da acerola (Malpighia punicifolia, L.) em diferentes fases de maturação. Revista Brasileira de Produtos Agroindustriais, v. 2, n. 2, p. 19-24, 2000.

[4] BENASSI, M.T.; ANTUNES, A.J. A comparison of metaphosphoric and oxalic acids as extractant solutions for the determination of vitamin $\mathrm{C}$ in selected vegetables. Arquivos de Biologia e Tecnologia, v. 31, n. 4, p. 507-513, 1988.

[5] CABRAL, A.C.D.; ORTIZ, S.A.; MADI, L.F.C.; SOLER, R.M.; GAZETA, E.F. Embalagem das frutas tropicais e seus produtos. In: Aspectos tecnológicos das frutas tropicais e seus produtos. Campinas: ITAL, (Série frutas tropicais), p. 199-296, 1980.

[6] EVAngelista, J. Tecnologia de alimentos. São Paulo: Atheneu, 1992, 652p.

[7] FIGUEIRÊDO, R.M.F. Caracterização físico-química do suco e pó de acerola (Malpighia punicifolia, L.). Campinas, 1998, 184p. Tese (Doutorado em Engenharia de Alimentos) - Universidade Estadual de Campinas (UNICAMP).

[8] GARCIA, E.E.C.; PADUlA, M.; SARANTÓPOUlOS, C.I.G.L. Embalagens plásticas: propriedades de barreira. Campinas: ITAL, 1989, 44p.

[9] GONZAGA NETO, L.; SOARES, J.M. Acerola para exportação: aspectos técnicos da produção. EMBRAPASPI, 1994, 43p.

[10] LIMA, M.F.M. Desidratação de polpa de umbu em leito de jorro: estudos fludodinâmicos e térmicos. Campina Grande. 1992, 188p. Dissertação (Mestrado em Engenharia Química), Universidade Federal da Paraíba.

[11] LIMA, M.L.; SANTOS, C.I.; COSTA, F.A.; LIMA, M.F.M.; SANTOS, E.M.B.D.; CARVALHO, L.P. Desidratação de Polpas de Frutas Tropicais em Leito de Jorro. In: CONGRESSO BRASILEIRO DE ENGENHARIA QUÍMICA. COBEQ - IC, Anais... São Carlos, 1995, p. 121-123.

[12] LOMAURO, C.J.; BAKSHI, A.S.; LABUZA, T.P. Evaluation of food moisture sorption isotherm equations. Part II: milk, coffee, tea, nuts, oilseeds, spices and starchy foods. Lebensmittel wissenchaft und Technology, v. 18, n. 1, p. 118-124, 1985.

[13] MATSUURA, F.C.A.U. Processamento de suco integral e concentrado de acerola. Campinas. 1994, 140p. Tese (Doutorado em Engenharia de Alimentos, Universidade Estadual de Campinas (UNICAMP).

[14] MEDEIROS, M.F.D. Influência da composição química dos materiais no desempenho do processo de secagem de polpas de frutas em leito de jorro. Campinas. 2001, 233p. Tese (Doutorado em Engenharia Química), Universidade Estadual de Campinas (UNICAMP).

[15] OLIVA, P.B.; MENEZES, H.C.; FERREIRA, V.L.P. Estudo da Estabilidade do Néctar de Acerola. Ciênc. Tecnol. Aliment., v. 16, n. 3, p. 228-232, 1996.

[16] PIERGIOVANNI, L. Materiais de embalagem e tecnologias de envase. In: BARUFFALDI, R.; OLIVEIRA, M.N. 
Fundamentos de Tecnologia de Alimentos. São Paulo: Atheneu, p. 219-278, 1998.

[17] PIMENTEL, L.M. Influência do processamento sobre a vitamina C do suco de acerola (M. glabra L.). Fortaleza. 1996, 85f. Dissertação (Mestrado em Tecnologia de Alimentos), Universidade Federal do Ceará.

[18] ROBBINS, J.A.; MOORE, P.P. Color change in fhesh red raspberry fruit stored at $0,4.5$, or $20^{\circ} \mathrm{C}$. HortScience, v. 25, n. 12, p. 1623-1624, 1990.
[19] SILVA, F.A.S.; AZEVEDO, C.A.V. Versão do programa computacional Assistat para o sistema operacional Windows. Revista Brasileira de Produtos Agroindustriais, v. 4, n. 1, p. 71-78, 2002.

[20] SOARES, E.C.; OLIVEIRA, G.S.F.; MAIA, G.A.; MONTEIRO, J.C.S.; SILVA, A.J.; FILHO, M.S.S. Desidratação da polpa de acerola (Malpighia emarginata D.C.). Ciênc. Tecnol. Aliment., v. 21, n. 2, p. 164$170,2001$. 